\title{
How public health policy can be informed by neuroscience
}

\author{
ANDREW B. WOLF \\ Department of Physiology and Biophysics, University of Colorado School of Medicine, USA \\ Medical Scientist Training Program, University of Colorado School of Medicine, USA \\ GIDON FELSEN * \\ Department of Physiology and Biophysics, University of Colorado School of Medicine, USA \\ Medical Scientist Training Program, University of Colorado School of Medicine, USA \\ Center for Bioethics and Humanities, University of Colorado Anschutz Medical Campus, USA
}

\begin{abstract}
Many public policies are designed to counteract commonly made decisions that result in poor health. These policies have primarily been informed by the behavioural economics of decision making. Underappreciated in this conversation has been the perspective from neuroscience, despite its recent success - and the likelihood of future progress in advancing our understanding of the neural basis for health-related decisions. Using tobacco control as an example, we provide a concise overview of how public health policies can and should be informed by neuroscience. We propose that such input can improve policies by increasing their effectiveness, improving screening efficiency and informing relevant ethical considerations. Finally, we recognise limitations and highlight roles that key stakeholders can play in incorporating neuroscientific evidence for the benefit of public policy.
\end{abstract}

Submitted 6 March 2017; accepted 28 August 2017

\section{Introduction}

From abusing substances to eating unhealthy food, people often make decisions that they know are deleterious to their health and well-being. Recently, policy makers have proposed using our increasing understanding of the psychology and cognitive biases underlying decisions (i.e. behavioural economics) (Kahneman, 2011) in order to implement strategies designed to improve the outcomes of such health-related decisions. We refer to such strategies as 'decisional enhancement' (DE) (Felsen et al., 2013), conceptually akin to cognitive

\footnotetext{
* Correspondence to: Department of Physiology and Biophysics, University of Colorado School of Medicine, 12800 E. 19th Ave., Aurora, CO 80045, USA. Email: gidon.felsen@ucdenver.edu
} 
(a)

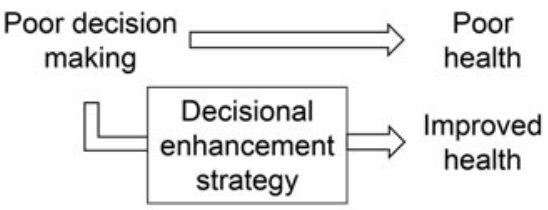

(b)

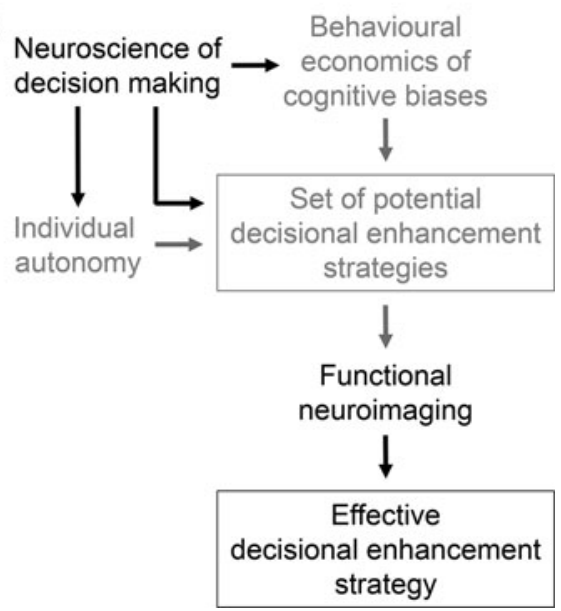

Figure 1. How neuroscience can improve health-promoting decisional enhancement strategies. (a) Decisional enhancement strategies leverage what is known about biases in decision making in order to promote decisions leading to improved health. (b) Potential decisional enhancement strategies have primarily been developed based on evidence from behavioural economics and informed, from an ethical perspective, based on their impact on individual autonomy (grey). We propose here that the neuroscience of decision making can increase the effectiveness of decisional enhancement strategies by enriching or complementing behavioural economics data and by informing the ethics of decisional enhancement strategies (black). In addition, functional neuroimaging can increase the efficiency of identifying those decisional enhancement strategies that are most likely to be effective (black).

enhancement (Farah et al., 2004) but with the goal of promoting better decision outcomes rather than increasing overall cognitive function (Figure 1(a)). DE strategies range from promoting top-down, rational reflection (e.g. waiting periods and information provision) to covertly counteracting cognitive biases (e.g. libertarian paternalism, or 'nudging') (Thaler \& Sunstein, 2008). Such strategies have been increasingly utilised in attempts to directly improve public health (Marteau et al., 2011; Marteau et al., 2012; Hansen et al., 2016), exemplified by former President Obama's inception of the Social and 
Behavioral Sciences Team in the USA following on the success of a similar initiative in the UK (Behavioural Insights Team, 2010). Underappreciated in the conversation, however, have been insights from neuroscience, despite the rapid recent advances - and future potential - in our understanding of the neural basis of decisions, including how and why we make particular healthrelated decisions. While there is substantial overlap between neuroscience and related disciplines like psychology and behavioural economics, our focus here is on those aspects of neuroscience that provide neurophysiologically based (rather than, for example, behaviourally based) explanatory power. There are multiple levels at which neurophysiology can be examined and understood, from intracellular processes to brain-wide metabolic activity; we consider this entire range of approaches as falling within the purview of 'neuroscience' as we use the term here. In this paper we propose several ways that such insights from neuroscience can improve public health DE strategies and recommend actions that stakeholders should take towards this end.

We illustrate our argument by focusing on DE strategies designed to reduce cigarette smoking (i.e. tobacco control) via graphic warning labels on cigarette packaging. Smoking represents an ideal target for DE strategies: despite years of public health campaigns and widespread knowledge of its profound health hazards, a stubborn $17 \%$ of US adults remain active smokers (Jamal et al., 2015), highlighting the insufficiency of policies utilised to date. We suggest that neuroscientific evidence and approaches can and should be used to improve tobacco control - and other health-promoting - DE strategies in three ways: by increasing their effectiveness, by improving screening efficiency and by informing their ethical considerations (Figure 1(b)). We describe each of these in turn, while also noting the limitations of neuroscience in this context, and conclude with some specific examples of how neuroscience can be incorporated into public policy.

\section{Increasing the effectiveness of decisional enhancement strategies}

Several types of tobacco control DE strategies based on the principles of behavioural economics have been attempted, with varying degrees of effectiveness (Loewenstein et al., 2012). As with many prevalent public health problems, even relatively small changes in behaviour (e.g. in usage rates) can have a large absolute impact on population health and societal cost. Thus, the more we understand about the neuroscience of decision making - whether it is considered to be complementary to, or to directly inform, behavioural economics (Jones, 2014; Grüne-Yanoff, 2016) - and, in particular, how the brain works in the context of addictive behaviours, the more we can tailor policy interventions to maximise effectiveness (Figure 1(b)) (Felsen \& Reiner, 2015). 
Evidence from human and animal model studies points to an important role for the medial prefrontal cortex in mediating emotional influences on decision making and in representing risk and reward (Euston et al., 2012). This region also exhibits aberrant activity during addiction-related behaviours, including during the response to nicotine exposure, suggesting its involvement in smoking-related decisions (Cohen \& George, 2013). These studies indicate that tobacco control strategies designed to modulate information processing in the medial prefrontal cortex, perhaps via eliciting emotional responses, may represent a promising approach. Indeed, warning labels that contain graphic images (e.g. a diseased lung) have been associated with thought processes linked to quitting cigarettes (e.g. perceived harm, self-reported desire to quit) (Noar et al., 2015), and several studies suggest that graphic warning labels decrease population-wide smoking activity (Azagba \& Sharaf, 2013; Jung, 2016) and increase quit attempts and successes for active smokers (Brewer et al., 2016).

A complementary strategy is suggested by evidence from neuroscience demonstrating an interesting quirk in how we update our beliefs in the face of new information. According to the 'optimism bias', individuals tend to change their beliefs more after being presented with favourable than unfavourable information (Sharot et al., 2011). Functional magnetic resonance imaging (fMRI) studies, which measure blood flow as a proxy for neural activity, have shown that this asymmetry occurs not during deliberation, but as soon as the information is presented (Sharot et al., 2011). As Tali Sharot has noted (Sharot, 2012), the timing of the neural response, as revealed by these fMRI studies, suggests that the information itself, rather than how it is integrated into existing beliefs, is critical in determining how the beliefs are updated. These results suggest that smokers may be more receptive to positive messages (e.g. ' $50 \%$ of smokers can quit') than negative messages (e.g. 'smokers die younger'), although this prediction remains to be tested. Increased dialogue between policy makers and neuroscientists would facilitate the integration of this sort of neuroscientific evidence - currently available and resulting from future studies - into the design of more effective DE strategies.

\section{Improving the efficiency of screening decisional enhancement strategies}

Once a set of potentially effective candidate DE strategies is designed, it is necessary to screen them to determine which will be most effective. Although the ultimate goal of such policies is, of course, to modify behaviour, testing multiple candidate policies at the behavioural level is inefficient. Functional neuroimaging offers the potential to streamline the process for determining which DE strategies are most likely to be effective (Figure 1(b)). Recent 
studies have shown that functional neuroimaging data provide additional predictive information, beyond self-reports and behavioural measures, about how individuals will respond to public health policies (Gabrieli et al., 2015). Thus, a large set of policies can be screened by obtaining such data from a representative sample of participants, and only those policies most likely to be successful would then need to be tested behaviourally. Importantly, the predictive power of neuroimaging data in this context is independent of whether the data address how a particular policy exerts its effect. Returning to the medial prefrontal cortex, recent work has demonstrated that activity in this region, as well as other regions associated with emotional memory, respond differently to graphic and neutral warning labels, providing physiological evidence that is complementary to self-reports of the emotional salience of the labels (Wang et al., 2015). These findings, in turn, may explain why activity in the medial prefrontal cortex can be used to predict the success of tobacco control campaigns (Falk et al., 2016). Thus, while inexpensive approaches can continue to be used to efficiently develop DE strategies (e.g. by examining public opinion via online surveys), neuroimaging screens can complement these approaches by providing unique insights into the mechanisms by which DE strategies may work (Whelan et al., 2017). Just as 'neuromarketers' have begun to use neuroimaging to efficiently identify effective advertising campaigns (Karmarkar \& Yoon, 2016), regulators charged with protecting consumers should consider a similar approach in order to identify effective DE strategies.

\section{Informing the ethics of decisional enhancement strategies}

The proposition that an individual's decision can be 'enhanced' raises immediate ethical questions about who is doing the enhancing and whether they have the individual's best interests in mind. We focus here on how neuroscience can inform one of the primary ethical objections to DE strategies, particularly to nudges operating below the level of conscious awareness: that they infringe upon individual autonomy (Figure 1(b)) (Bovens, 2009; Blumenthal-Barby \& Burroughs, 2012; Grüne-Yanoff, 2012; White, 2013; Sunstein, 2015) (note that we do not consider here other important ethical issues relevant to DE strategies, such as justice or societal equity). Briefly, we suggest that evidence from neuroscience can inform this ethical concern about autonomy in two ways.

First, it can illuminate our understanding of autonomy directly, in ways that are relevant to the concern over autonomy infringement. In particular, in support of behavioural studies, neuroscience has called into question the extent to which many of our everyday decisions are sufficiently free of undue external influence to be considered autonomous in the traditional individualist sense (Custers \& Aarts, 2010; Felsen \& Reiner, 2011; Bode et al., 2014). To 
take just one example, activity in regions known to be activated by fear underlies aversion to losses, and does so covertly (De Martino et al., 2006; Tom et al., 2007). Instead, the evidence from neuroscience is more consistent with relational conceptions of autonomy that recognise that our decisions are, by nature, influenced by a variety of sources (Christman, 2009; Felsen \& Reiner, 2011). In this case, a tobacco control DE strategy (e.g. a graphic warning label) simply provides yet one more influence on a smoking-related decision that is already influenced by numerous other sources (e.g. advertisements associating smoking with attractiveness). Thus, while the influence of DE strategies might appear to infringe upon autonomy as described by individualistic accounts, seen in the light of relational autonomy - with which the neuroscientific evidence is more consistent - these influences appears no more ethically problematic than the advertising campaigns that they are designed to counteract.

Second, neuroscience can address autonomy-related concerns about DE strategies by explicating the mechanisms by which the strategies themselves may influence decision making. In particular, some DE strategies are designed to elicit decisions consistent with 'higher-order desires' (e.g. staying healthy) (Frankfurt, 1971; Dworkin, 1988) by counteracting detrimental internally generated biases (e.g. biological cravings), and therefore may promote, rather than infringe upon, autonomy. Although the ethics of influencing decisions is complex, accounting for the perspective from neuroscience would allow the impact of DE strategies on autonomy to be more comprehensively considered.

\section{Limitations of neuroscience evidence for informing decisional enhancement strategies}

Our goal in this paper is to explain how neuroscience can complement other disciplines in informing policies designed to improve decision making. It is also important, however, to note several limitations of using neuroscientific evidence for this purpose. First, while rapid advances in neuroscience hold great promise for informing policy in the near future, the real impact of the field is currently limited by how much we still do not understand about fundamental brain function, complicating translation to the policy domain (Seymour \& Vlaev, 2012). It is therefore critical that the current value of neuroscience for policy not be overstated, particularly since neuroscientific evidence is often particularly persuasive, even if it is not entirely understood (Weisberg et al., 2007; O'Connor et al., 2012). In addition, cost-effectiveness should be considered: it would be impractical to replace less expensive means of data collection with costly fMRI experiments. Finally, while we have focused here on the benefits of neuroscience for informing DE strategies, we recognise that the ultimate 
goal of such strategies is to modify behaviour. Thus, we do not suggest that neuroscience can or should replace behavioural experiments, which will remain critical for validating DE strategies.

\section{Conclusions}

Given advances in our understanding of decision making, across multiple levels (Gold \& Shadlen, 2007; Heekeren et al., 2008; Levy \& Glimcher, 2012), we suggest that neuroscience is poised to make unique contributions to improving the design and evaluation of DE strategies. These policy improvements can be realised in several ways; we focus on the potential roles of US agencies as examples, but our arguments apply to any country with analogous agencies. First, interdisciplinary forums at which neuroscientists can collaborate with policy makers to design efficient and ethical DE strategies should be fostered and promoted. Input from neuroscience would be valuable to the ground-breaking work of efforts like those of the Social and Behavioral Sciences Team, especially given the increasing pace of relevant research. Second, functional neuroimaging can be used by regulators like the Food and Drug Administration (FDA) and Federal Trade Commission to more efficiently identify effective strategies. Third, policies can be improved via increased funding investment from agencies like the National Institutes of Health (NIH) and Centers for Disease Control for research on translating our understanding of brain function to the policy domain. The Tobacco Centers of Regulatory Science programme, supported by the NIH and the FDA, provides a model for how policy-relevant research can be stimulated (Berman \& Kim, 2015). Given the high costs of treatments resulting from unhealthy choices, such funding would appear to be a wise investment, reducing spending in the long term.

While we have focused here on DE strategies related to cigarette smoking, we suggest that our framework may apply to policies designed to influence healthrelated behaviours more broadly (although many of the specific findings we have discussed may be applicable only to smoking-related behaviour). Most directly, the accelerating trend towards marijuana legalisation presents similar public health challenges as does tobacco use. Obesity may represent an analogous case: like smoking, it is costly to society and is associated with a rapidly growing neuroscientific literature exploring its physiology. Early efforts exist to translate evidence from neuroscience about obesity to DE strategies, such as the European Commission-funded Nudge-it (www.nudge-it.eu).

Smart public health policies for improving our predictably poor healthrelated decisions (Ariely, 2008) appear to be here to stay. We hope to have shown here that, as progress in illuminating brain function accelerates, these policies can and should be increasingly informed by neuroscience. 


\section{References}

Ariely, D. (2008), Predictably Irrational: The hidden forces that shape our decisions, New York: Harper Press.

Azagba, S. and M. F. Sharaf (2013), 'The effect of graphic cigarette warning labels on smoking behavior: evidence from the Canadian experience', Nicotine \& Tobacco Research, 15(3): 708-717.

Behavioural Insights Team. (2010), Applying behavioural insight to health. UK Cabinet Office. Available from: https:/www.gov.uk/government/publications/applying-behavioural-insightto-health-behavioural-insights-team-paper

Berman, M. L. and A. E. Kim (2015), 'Bridging the gap between science and law : the example of tobacco regulatory science', Journal of Law, Medicine, \& Ethics, 43(s1): 95-98.

Blumenthal-Barby, J. S. and H. Burroughs (2012), 'Seeking better health care outcomes: the ethics of using the "nudge", The American Journal of Bioethics, 12(2): 1-10.

Bode, S., C. Murawski, C. S. Soon, P. Bode, J. Stahl and P. L. Smith (2014), 'Demystifying "free will”: The role of contextual information and evidence accumulation for predictive brain activity', Neuroscience \& Biobehavioral Reviews, 47: 636-645.

Bovens, L. (2009), 'The Ethics of Nudge', in T. Grüne-Yanoff and S. O. Hansson (eds), Preference Change, Volume 42, Netherlands: Springer, 207-219.

Brewer, N. T., M. G. Hall, S. M. Noar, H. Parada, A. Stein-Seroussi, L. E. Bach, S. Hanley and K. M. Ribisl (2016), 'Effect of pictorial cigarette pack warnings on changes in smoking behavior: a randomized clinical trial', JAMA Internal Medicine, 176(7): 905-912.

Christman, J. (2009), The Politics of Persons: Individual Autonomy and Socio-historical Selves, Cambridge: Cambridge University Press.

Cohen, A. and O. George (2013), 'Animal models of nicotine exposure: relevance to second-hand smoking, electronic cigarette use, and compulsive smoking', Frontiers in Psychiatry, 4: 41.

Custers, R. and H. Aarts (2010), 'The unconscious will: how the pursuit of goals operates outside of conscious awareness', Science, 329(5987): 47-50.

De Martino, B., D. Kumaran, B. Seymour and R. J. Dolan (2006), 'Frames, biases, and rational decision-making in the human brain', Science, 313(5787): 684-687.

Dworkin, G. (1988), The Theory and Practice of Autonomy, Cambridge: Cambridge University Press.

Euston, D. R., A. J. Gruber and B. L. McNaughton (2012), 'The role of medial prefrontal cortex in memory and decision making', Neuron, 76(6): 1057-1070.

Falk, E. B., M. B. O’Donnell, S. Tompson, R. Gonzalez, S. D. Cin, V. Strecher, K. M. Cummings and L. An (2016), 'Functional brain imaging predicts public health campaign success', Social Cognitive and Affective Neuroscience, 11(2): 204-214.

Farah, M. J., J. Illes, R. Cook-Deegan, H. Gardner, E. Kandel, P. King, E. Parens, B. Sahakian and P. R. Wolpe (2004), 'Neurocognitive enhancement: what can we do and what should we do?', Nature Reviews Neuroscience, 5(5): 421-425.

Felsen, G., N. Castelo and P. B. Reiner (2013), 'Decisional enhancement and autonomy: public attitudes towards overt and covert nudges', Judgment and Decision Making, 8(3): 202-213.

Felsen, G. and P. B. Reiner (2011), 'How the neuroscience of decision making informs our conception of autonomy', The American Journal of Bioethics - Neuroscience, 2(3): 3-14.

Felsen, G. and P. B. Reiner (2015), 'What can neuroscience contribute to the debate over nudging?', Review of Philosophy and Psychology, 6(3): 469-479.

Frankfurt, H. G. (1971), 'Freedom of the Will and the Concept of a Person', The Journal of Philosophy, 68(1): 5-20.

Gabrieli, J. D. E., S. S. Ghosh and S. Whitfield-Gabrieli (2015), 'Prediction as a humanitarian and pragmatic contribution from human cognitive neuroscience', Neuron, 85(1): 11-26. 
Gold, J. I. and M. N. Shadlen (2007), 'The neural basis of decision making', Annual Review of Neuroscience, 30: 535-74.

Grüne-Yanoff, T. (2012), 'Old wine in new casks: libertarian paternalism still violates liberal principles', Social Choice and Welfare, 38(4): 635-645.

Grüne-Yanoff, T. (2016), 'Why behavioural policy needs mechanistic evidence', Economics \& Philosophy, 32(3): 463-483.

Hansen, P. G., L. R. Skov and K. L. Skov (2016), 'Making healthy choices easier: regulation versus nudging', Annual Review of Public Health, 37(1): 237-251.

Heekeren, H. R., S. Marrett and L. G. Ungerleider (2008), 'The neural systems that mediate human perceptual decision making', Nature Reviews Neuroscience, 9(6): 467-479.

Jamal, A., D. M. Homa, E. O'Connor, S. B. Babb, R. S. Caraballo, T. Singh, S. S. Hu and B. A. King (2015), 'Current cigarette smoking among adults — United States, 2005-2014', Morbidity and Mortality Weekly Report, 64(44): 1233-1240.

Jones, O. D. (2014), 'Why behavioral economics isn't better, and how it could be'. Available from: https://papers.ssrn.com/sol3/papers.cfm?abstract_id=2504776

Jung, M. (2016), 'Implications of graphic cigarette warning labels on smoking behavior: an international perspective', Journal of Cancer Prevention, 21(1): 21-25.

Kahneman, D. (2011), Thinking, Fast and Slow, New York: Farrar, Straus, and Giroux.

Karmarkar, U. R. and C. Yoon (2016), 'Consumer neuroscience: advances in understanding consumer psychology', Current Opinion in Psychology, 10: 160-165.

Levy, D. J. and P. W. Glimcher (2012), 'The root of all value: a neural common currency for choice', Current Opinion in Neurobiology, 22(6): 1027-1038.

Loewenstein, G., D. A. Asch, J. Y. Friedman, L. A. Melichar and K. G. Volpp (2012), 'Can behavioural economics make us healthier?', BMJ, 344: e3482.

Marteau, T. M., D. Ogilvie, M. Roland, M. Suhrcke and M. P. Kelly (2011), 'Judging nudging: can nudging improve population health?', BMJ, 342(3): 263-265.

Marteau, Theresa M., G. J. Hollands and P. C. Fletcher (2012), 'Changing human behavior to prevent disease: the importance of targeting automatic processes', Science, 337(6101): 1492-1495.

Noar, S. M., M. G. Hall, D. B. Francis, K. M. Ribisl, J. K. Pepper and N. T. Brewer (2015), 'Pictorial cigarette pack warnings: a meta-analysis of experimental studies', Tobacco Control, 25(3): 341-354.

O'Connor, C., G. Rees and H. Joffe (2012), 'Neuroscience in the public sphere', Neuron, 74(2): 220-226.

Seymour, B. and I. Vlaev (2012), 'Can, and should, behavioural neuroscience influence public policy?', Trends in Cognitive Sciences, 16(9): 449-451.

Sharot, T. (2012), The optimism bias (Presentation). Available from: https://www.ted.com/talks/ tali_sharot_the_optimism_bias

Sharot, T., C. W. Korn and R. J. Dolan (2011), 'How unrealistic optimism is maintained in the face of reality', Nature Neuroscience, 14(11): 1475-1479.

Sunstein, C. R. (2015), 'The Ethics of Nudging', Yale Journal on Regulation, 32(3): 413-450.

Thaler, R. H. and C. R. Sunstein (2008), Nudge: Improving decisions about health, wealth, and happiness, New Haven: Yale University Press.

Tom, S. M., C. R. Fox, C. Trepel and R. A. Poldrack (2007), 'The neural basis of loss aversion in decision-making under risk', Science, 315(5811): 515-518.

Wang, A.-L., S. B. Lowen, D. Romer, M. Giorno and D. D. Langleben (2015), 'Emotional reaction facilitates the brain and behavioural impact of graphic cigarette warning labels in smokers', Tobacco Control, 24(3): 225-232.

Weisberg, D. S., F. C. Keil, J. Goodstein, E. Rawson and J. R. Gray (2007), 'The seductive allure of neuroscience explanations', Journal of Cognitive Neuroscience, 20(3): 470-477. 
46 ANDREW B. WOLF AND GIDON FELSEN

Whelan, M. E., P. S. Morgan, L. B. Sherar, M. W. Orme and D. W. Esliger (2017), 'Can functional magnetic resonance imaging studies help with the optimization of health messaging for lifestyle behavior change? A systematic review', Preventive Medicine, 99: 185-196.

White, M. D. (2013), The Manipulation of Choice: Ethics and Libertarian Paternalism, Palgrave Macmillan. 